\title{
GEOMETRIC THEORY OF DIFFERENTIAL EQUATIONS. THE LJAPUNOV INTEGRAL FOR MONOTONE COEFFICIENTS
}

\author{
BY H. GUGGENHEIMER ${ }^{1}$
}

Communicated by James Serrin, March 3, 1971

An equation

$$
x^{\prime \prime}+p(t) x=0, \quad-\infty<t<\infty, p(t)>0,
$$

can be considered as the Frenet equation of a locally convex curve $x(t)=\left(x_{1}(t), x_{2}(t)\right)$ in unimodular centroaffine differential geometry [1]. The osculating ellipse $E_{u}$ at $x(u)$ is the solution of

$$
y^{\prime \prime}+p(u) y=0, \quad y(u)=x(u), \quad y^{\prime}(u)=x^{\prime}(u) .
$$

We prove an unimodular centroaffine Kneser theorem:

THEOREM 1. If $p(t)$ is strictly monotone and differentiable in an interval $[a, b]$, then every osculating ellipse $E_{t}, t \in[a, b]$, contains all osculating ellipses of smaller area defined on the same interval in its interior.

The area of the osculating ellipse is proportional to $p(t)^{-1 / 2}$. By the Jordan curve theorem, the assertion is true if it is true for neighboring points. Then it is easily checked that a pair of conjugate diameters of the smaller ellipse is in the interior of the larger one. The approximation and convergence theorems of convexity imply:

THEOREM 2. If $p(t)$ is monotone and continuous in $[a, b]$, then every osculating ellipse $E_{t}, t \in[a, b]$, contains all osculating ellipses of smaller area defined on the same interval.

The parameter $t-u$ is equal to two times the area covered by the radius vector of $x(t)$ and $\int_{u}^{t} p(\tau) d \tau$ is equal to two times the area covered by the radius vector of the polar reciprocal $x^{*}(t)$ of $x$ for the unit circle, if $x(t)$ is a curve of unit Wronskian $[1, \S 3]$. For $p$ monotone increasing, the curve $x$ and the osculating ellipses $E_{t}(t \geqq u)$ are contained in $E_{u}$, and $x^{*}(\tau), E_{\tau}^{*}(u \leqq \tau \leqq t)$ are in $E_{t}^{*}$.

Let $\phi(u)$ be the conjugate point of $u$ for (1), i.e., the zero following

AMS 1970 subject classifications. Primary 34B05, 53A40, 34C10; Secondary 34A40, 53A15.

Key words and phrases. Unimodular centroaffine differential geometry, Kneser theorem, Ljapunov integral, conjugate point, co-conjugate point.

1 Research partially supported by NSF Grant No. GP-19133. 
$u$ of a nontrivial solution of (1) that vanishes at $u$. Let $\psi(u)$ be the co-conjugate point of $u$ for (1), i.e., the zero following $u$ of the derivative of a nontrivial solution of (1) whose derivative vanishes at $u$. A major topic in the study of equations (1) are estimates of the Ljapunov integral

$$
L(u)=[\phi(u)-u] \int_{u}^{\phi(u)} p(t) d t .
$$

We suppose that $p(t)$ is monotone increasing and continuous and that $\phi(u)<\infty$. As an application of Theorem 2, we have

$$
\begin{gathered}
\pi p(\phi(u))^{-1 / 2} \leqq \phi(u)-u \leqq \pi p(u)^{-1 / 2}, \\
\pi p(u)^{1 / 2} \leqq \int_{u}^{\phi(u)} p(t) d t .
\end{gathered}
$$

If $\psi(u) \geqq \phi(u)$, then

$$
\int_{u}^{\phi(u)} p(t) d t \leqq \pi p(\phi(u))^{1 / 2} .
$$

If $\psi(u)<\phi(u)$, then

$$
\int_{u}^{\phi(u)} p(t) d t \leqq \frac{3}{2} \pi p(\phi(u))^{1 / 2} .
$$

(The difference $\phi(u)-\psi(u)$ has been investigated in [2].) Together, we obtain:

THEOREM 3. For monotone increasing, continuous, positive $p(t)$, the Ljapunov integral satisfies

$$
\begin{gathered}
\pi^{2}\left(\frac{p(u)}{p(\phi(u))}\right)^{1 / 2} \leqq L(u) \leqq\left[1+\frac{1+\epsilon}{4}\right] \pi^{2}\left(\frac{p(\phi(u))}{p(u)}\right)^{1 / 2}, \\
\epsilon=\operatorname{sgn}[\phi(u)-\psi(u)] . \\
\text { REFERENCES }
\end{gathered}
$$

1. H. Guggenheimer, Hill equations with coexisting periodic solutions, J. Differential Equations 5 (1969), 159-166. MR 39 \#550.

2. - Geometric theory of differential equations, III. Second order equations on the reals, Arch. Rational Mech. Anal. (to appear).

Polytechnic Institute of Brooklyn, Brooklyn, New York 11201 